\title{
Aberration Free Microscopy for Life Science Applications
}

\author{
J. M. Plitzko*, B. Freitag**, R. Hegerl* and U. Lücken** \\ * Max-Planck-Institute of Biochemistry, Molecular Structural Biology, Am Klopferspitz 18, \\ 82152 Martinsried, Germany \\ ** FEI Company, Achtseweg Noord 5, P.O. Box 80066, 5600 KA Eindhoven, The Netherlands
}

With the advent of aberration correctors, electron monochromators and improved energy filters for the newest generation of transmission electron microscopes (TEMs), disciplines like material science and solid state physics already demonstrated the radically improved image quality combined with the supreme spatial resolution for structural characterization [1-5]. This technical capability clearly revolutionized the field of electron microscopy during the last years in its various flavours, e.g. SEM [6], TEM [5] and STEM [2]. However, the consideration of aberration free microscopy to improve biological imaging, e.g. by means of cryo-electron microscopy (cryo-EM) has never been reported so far. In our study we have explored the possibilities of a $\mathrm{C}_{\mathrm{s}}$-corrected TEM for investigations on biological samples.

For our experiments we have chosen the $\mathrm{C}_{\mathrm{s}}$-corrected FEI Tecnai F20 ST operating with a field emission gun (FEG) at $200 \mathrm{kV}$ acceleration voltage, equipped with an $\mathrm{C}_{\mathrm{s}}$-corrector [5] and a Gatan $1 \mathrm{kx} 1 \mathrm{k}$ CCD camera. Moreover, special cryo-shields were installed to enable investigations of vitrified samples at liquid nitrogen temperature.

We have selected the structurally well-known proteasome $20 \mathrm{~S}(721 \mathrm{kDa},[7])$ and VAT (valosine containing protein-like ATPase, $500 \mathrm{kDa}$, [8]) from the archaeon Thermoplasma acidophilum. Both samples were applied to a continuous carbon film and negatively stained with uranyl acetate. Furthermore unstained ice-embedded proteasome 20S specimens have been prepared on Quantifoil grids and used for the $\mathrm{C}_{\mathrm{s}}$-corrected cryo-EM experiments.

Modern microscopes reach resolution levels better than $1 \AA$, which is more than sufficient for work in structural biology. However, the highest instrumental resolution is reached close to 'Scherzer focus', but at such defocus values the transfer of the low frequency information is extremely poor for cryo-EM studies of single molecules. On the other hand the low frequency information is essential for locating the particles within an electron micrograph. Close to 'Scherzer' the noisy particles are no longer discernible against the background and thus they are simply invisible (Fig. 1E). Therefore, large defocus values up to $3 \mu \mathrm{m}$ or more have to be used with the consequence that the contrast transfer function (CTF) oscillates many times in the high frequency domain. Moreover these oscillations lead to a 'spreading' (point spread function, PSF) of the information within the image, resulting in ,blurred' images, which in turn requires a CTF correction of the recorded images $[9,10]$. This effect of contrast delocalization, especially known from FEG instruments increases with increasing $\mathrm{C}_{\mathrm{s}}$ [11]. Here, spherical-aberration correction offer some remedy (Fig. 1A). The adverse contribution ('blurring') of delocalized electron waves can be removed and contrast is improved as already shown in material science studies $[4,11]$.

However with decreasing $\mathrm{C}_{\mathrm{s}}$ the contrast, especially in the low spatial frequency range will decrease as well, which can be observed, e.g. going from BioTwin lense pole piece designs $\left(\mathrm{C}_{\mathrm{s}} \sim 6 \mathrm{~mm}\right)$ to commonly used Twin $\left(\mathrm{C}_{\mathrm{s}} \sim 2 \mathrm{~mm}\right)$ or even to SuperTwin objective lens systems $\left(\mathrm{C}_{\mathrm{s}} \sim 1.2 \mathrm{~mm}\right)$. The question arises: What can be expected from $\mathrm{C}_{\mathrm{s}}$-correction for the investigation of biological samples (especially in cryo-EM studies, where large defocus values are mandatory)? 
In the course of our study we have investigated the influence of spherical-aberration correction on negatively stained samples at different defocus settings and at different $\mathrm{C}_{\mathrm{s}}$-corrector settings (Fig . 1 $B, C, D$ ), namely we adjusted negative as well as positive $C_{s}$-values (in the range of a couple of 100 $\mu \mathrm{m})$ and compared them to a aberration free acquisition. Moreover we will report on the first $\mathrm{C}_{\mathrm{s}^{-}}$ corrected cryo-EM experiments on vitrified samples at different defocii (Fig. 1E,F,G). The results and the possible outcome of this study are still subject to an in-depth image analysis and we will present and critically discuss our most recent findings.

\section{References}

[1] C. J. Jia et al., Science 299, 870-873 (2003)

[2] P. E. Batson et al., Nature 418, 617-620 (2002)

[3] B. Kabius et al., Journal of Electron Microscopy 51 (suppl.), 551-558 (2002)

[4] M. Lentzen et al., Ultramicroscopy 92, 233-242 (2002)

[5] M. Haider et al., Nature 392, 768-769 (1998)

[6] J. Zach and M. Haider, Optik 98, 112-118 (1995)

[7] W. Baumeister et al., Cell 92 (3), 367-380 (1998)

[8] B. Rockel et al., Journal of Molecular Biology 317, 673-681 (2002)

[9] M. van Heel et al., Quarterly Reviews of Biophysics 33 (4), 307-369 (2000)

[10] B. Sander et al., Journal of Structural Biology 142, 392-401 (2003)

[11] M. Haider et al., Ultramicroscopy 75, 53-60 (1998)

[12] We thank the European Union for financial support within the 3DEM network of excellence (NoE). Moreover we would like to thank H. Lichte from the Technical University in Dresden, Germany for the permission to use his microscope. We are grateful to H. Uland for technical support and O. Mihalache and B. Kühlmorgen for their help with sample preparation.
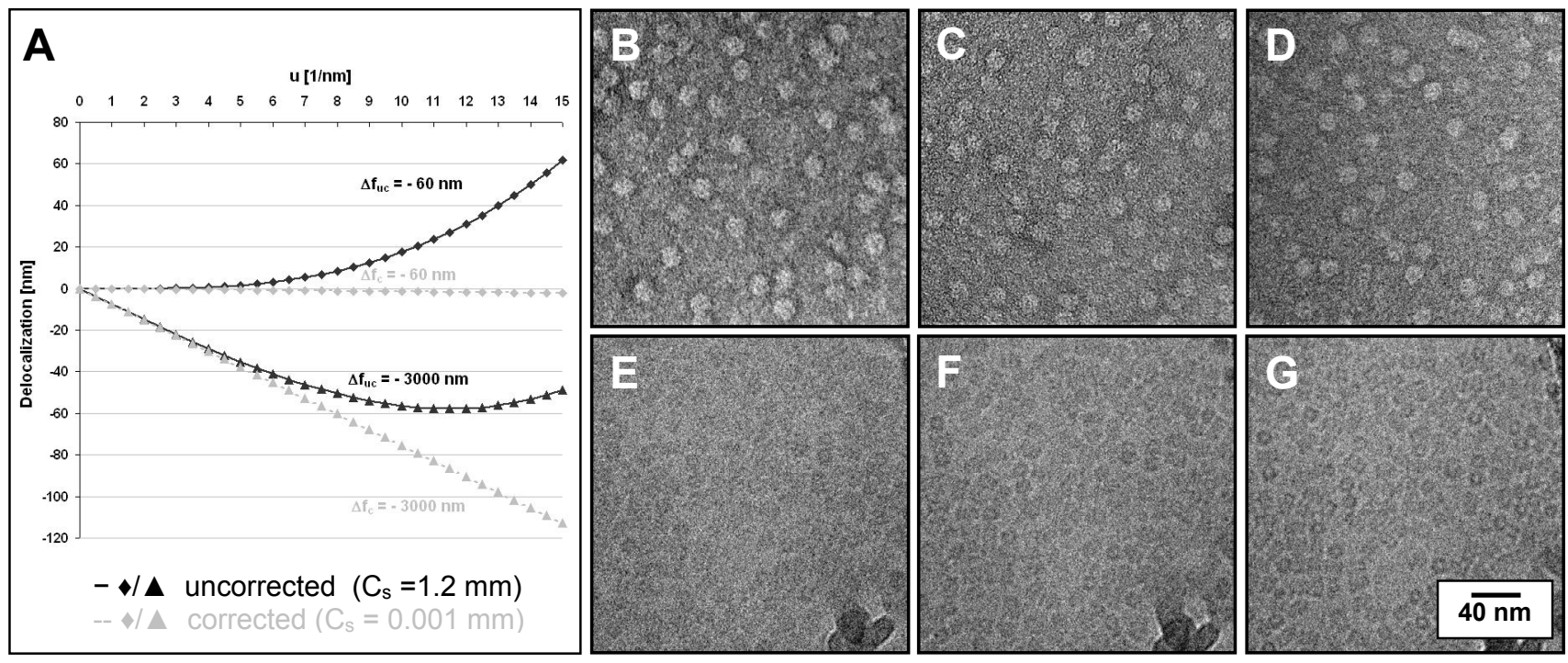

Figure 1: (A) Delocalization calculation for different $\mathrm{C}_{\mathrm{s}^{-}}$and defocus settings. Black solid lines for the uncorrected case with a $C_{\mathrm{s}}=1.2 \mathrm{~mm}, \Delta \mathrm{f}_{\mathrm{uc}}=-60 \mathrm{~nm}$ ('Scherzer') and $\Delta \mathrm{f}_{\mathrm{uc}}=-3000 \mathrm{~nm}$ and grey dashed lines for $\mathrm{C}_{\mathrm{s}}=0.001 \mathrm{~mm}$ for $\Delta \mathrm{f}_{\mathrm{c}}=-60 \mathrm{~nm}$ ('Scherzer') and $\Delta \mathrm{f}_{\mathrm{c}}=-3000 \mathrm{~nm}$.

First row of images: Negatively stained VAT single macromolecules close to 'Scherzer focus' at different $\mathrm{C}_{\mathrm{s}}$-corrector settings. (B) $\mathrm{C}_{\mathrm{s}} \sim 1.2 \mathrm{~mm}$ (C) $\mathrm{C}_{\mathrm{s}} \sim+150 \mu \mathrm{m}$ and (D) $\mathrm{C}_{\mathrm{s}} \sim-200 \mu \mathrm{m}$. Second row of images: Spherical aberration corrected cryo-EM images of the proteasome $20 \mathrm{~S}$ embedded in vitrified ice at different defocus settings $(\mathrm{Cs} \sim 10 \mu \mathrm{m})$. (E) close to 'Scherzer', (F) $\Delta \mathrm{f} \sim-2 \mu \mathrm{m}$ and (G) at $\Delta \mathrm{f} \sim-4 \mu \mathrm{m}$. 